\title{
ON THE CONNECTIVITY AND DIAMETER OF SMALL-WORLD NETWORKS
}

\author{
AYALVADI GANESH, ${ }^{*}$ Microsoft Research \\ FENG XUE, ${ }^{* *}$ University of Illinois at Urbana-Champaign
}

\begin{abstract}
We consider two different models of small-world graphs on nodes whose locations are modelled by a stochastic point process. In the first model each node is connected to a fixed number of its nearest neighbours, while in the second model each node is connected to all nodes located within some fixed distance. In both models, nodes are additionally connected via shortcuts to other nodes chosen uniformly at random. We obtain sufficient conditions for connectivity in the first model, and necessary conditions in the second model. Thereby, we show that connectivity is achieved at a smaller value of total degree (nearest neighbours plus shortcuts) in the first model. We also obtain bounds on the diameter of the graph in this model.
\end{abstract}

Keywords: Random graph; small-world model; connectivity

2000 Mathematics Subject Classification: Primary 05C80

\section{Introduction}

A classical random graph model introduced by Erdős and Rényi [5] consists of $n$ nodes, with the edge between each pair of nodes being present with probability $p_{n}$, independent of all other edges. This model, which is known as the Bernoulli random graph model, has been studied extensively, and many of its properties are well understood. For instance, Erdôs and Rényi showed that this random graph model exhibits a sharp threshold for connectivity at $p_{n}=\log n / n$. Precisely, if $n p_{n}-\log n \rightarrow c$ as $n \rightarrow \infty$ for some constant $c$, then the probability that the graph is connected goes to $\exp \left(-\mathrm{e}^{-c}\right)$. Note that $(n-1) p_{n}$ is the expected node degree, so the result states that there is a sharp threshold for connectivity at an expected node degree of $\log n$. A number of variants of the above model, such as random regular graphs, have also been studied extensively. A feature common to these models is that the nodes are exchangeable.

Recently, there has been considerable interest in a different class of models, namely, spatial or geometric random graphs; see, for example, [9] and [11]. Here, the nodes are associated with coordinates in a Euclidean space, and the probability of an edge between a pair of nodes is typically some function of the distance between them (or, more generally, of their spatial coordinates). The node positions are also the realisation of some random process. One example is obtained by placing $n$ nodes uniformly on the unit square and putting an edge between

Received 24 January 2005; revision received 31 August 2007.

* Current address: Department of Mathematics, University of Bristol, University Walk, Bristol BS8 1TW, UK.

Email address: a.ganesh@bristol.ac.uk

** Current address: Communications Technology Laboratory, Intel Corporation, Santa Clara, CA 95052, USA.

Email address: feng.xue1@intel.com 
any pair of nodes if the distance between them is smaller than a threshold $r_{n}$. Penrose [10] showed that the probability of connectivity goes to 0 if $\pi n r_{n}^{2}-\log n \rightarrow-\infty$, and goes to 1 if $\pi n r_{n}^{2}-\log n \rightarrow+\infty$. Similar results were also obtained by Gupta and Kumar [6] in the context of a model of wireless networks.

Since $\pi n r_{n}^{2}$ is the expected degree of each node (except near the edges of the square), we see that there is a threshold for connectivity at a mean degree of $\log n$, which is the same as in the Bernoulli random graph. A somewhat different model was studied in Xue and Kumar [13]. Here, each node is connected to its $m_{n}$ nearest neighbours; more precisely, the edge between $u$ and $v$ is present if either $u$ is one of the $m_{n}$ nodes closest to $v$ or $v$ is one of the $m_{n}$ nodes closest to $u$. For this model, the authors show that the probability of connectivity goes to 1 if $m_{n}>5.1774 \log n$ and to 0 if $m_{n}<0.074 \log n$. These results were improved by Balister et al. [1] who showed that $m_{n} \geq 0.3043 \log n$ is necessary and $m_{n}>0.5139 \log n$ is sufficient for connectivity (with the probability going to 1 as $n$ tends to $\infty$ ). It is not known whether there is a threshold for the connectivity at $c \log n$ for some constant $c$. We remark that in the above models the same results hold if we consider a Poisson point process of intensity $n$ instead of $n$ points uniformly distributed on the unit square.

One of the motivations for interest in spatial random graphs is their applicability to wireless communication networks [6], [13]. Spatial random graphs on high-dimensional spaces might offer good models for social networks, which are poorly described by Bernoulli random graphs. Another class of models that has attracted attention in the latter context are so-called 'smallworld networks'. One commonly used way to model such networks is to consider nodes as located at the points of a (finite or infinite) $d$-dimensional lattice, and to augment the lattice with shortcuts, which are additional edges between pairs of nodes. The shortcut between a pair of nodes is present with a probability that is typically some function of the distance between them. Since the lattice is already connected, interest in these models has focused on how the diameter is reduced by the presence of shortcuts (see, for example, [2], [4], and [12, pp. 66-70]), and also on whether efficient decentralised routeing is possible [7].

In this paper we consider two variants of the above small-world model. We model node locations by a stochastic point process, e.g. independent and identically distributed uniformly on a square. Nodes are connected by nearest neighbour links, either to a fixed number of nodes closest to them, or to all nodes within a fixed distance. In addition, nodes are joined by shortcuts to other nodes chosen uniformly at random. We are interested in how connectivity depends on the number of nearest neighbours and the number of shortcuts. In the next section we address this question after providing a precise definition of the models. We also obtain bounds on the graph diameter of the small-world network in the connected regime.

\section{Main results}

We consider two different models of a small-world network, denoted Model A and Model B. In each case we consider a sequence of random networks $G_{n}$ indexed by a parameter $n \in \mathbb{N}$, which we call the size of the network. We say that a property $Q$ holds with high probability (w.h.p.) if $\mathrm{P}\left(G_{n}\right.$ possesses the property $\left.Q\right)$ goes to 1 as $n$ tends to $\infty$. In all cases we consider undirected graphs.

Model A. There are $n$ nodes, and each node chooses $m_{n}$ other nodes to connect to, called its nearest neighbours. In addition, a shortcut is present between each pair of nodes with probability $p_{n}$, independently of all other edges. (If two nodes are connected by both a nearest neighbour edge and a shortcut, we replace the multiple edge by a simple one.) 
Note that the 'nearest neighbour' relation need not be symmetric. An edge is present between nodes $x$ and $y$ if either $y$ is one of the $m_{n}$ nearest neighbours of $x$ or $x$ is one of the $m_{n}$ nearest neighbours of $y$ or there is a shortcut between them. The model is parametrised by the sequences $m_{n}$ and $p_{n}$, and shortcuts are the only source of randomness in this model. The terminology of 'nearest neighbour' may be misleading: as far as our results, below, are concerned, it only matters that each node connects to $m_{n}$ other nodes, chosen arbitrarily. However, we have chosen to use this term for concreteness, and because it was motivated by applications.

Example. Suppose that the nodes are located uniformly at random on the unit square. In this case Model A incorporates elements of both Bernoulli random graphs and the Xue-Kumar model, and our results, stated in Theorem 1, below, apply to every realisation of the node locations.

Model B. There are $n$ nodes, located uniformly at random on the torus obtained by identifying the opposite sides of the square $[-\sqrt{n} / 2, \sqrt{n} / 2]^{2}$ of area $n$ centred at the origin. Each node is connected to all nodes within a radius $r_{n}$ and, in addition, shortcuts are present between each pair of nodes with probability $p_{n}$, independent of all other edges.

Model B is parametrised by the sequences $r_{n}$ and $p_{n}$. It combines elements of Bernoulli and spatial random graphs. Observe that the shortcut distribution is the same in Models A and B. Thus, the main difference between the models is that the number of nearest neighbours is random in Model B but deterministically bounded below in Model A. We shall see that this greatly improves connectivity in Model A.

We consider a sequence of random graphs indexed by $n$. We denote by $C_{n}$ the event that the $n$th random graph is connected. We denote by $D_{n}$ the diameter of the graph, namely, the maximum over all node pairs of the length of the shortest path between them, in terms of the number of edges. We take $D_{n}=\infty$ if the graph is not connected.

Theorem 1. Suppose that the sequences $m_{n}$ and $p_{n}$ are such that

$$
\begin{gathered}
\frac{m_{n}}{n} \rightarrow 0 \text { as } n \rightarrow \infty, \text { and } \\
\left(m_{n}+1\right) n p_{n}>2(1+\delta) \log \frac{n}{m_{n}+1}
\end{gathered}
$$

for some $\delta>0$ and all sufficiently large $n$. Then, for the random graph described in Model A, above, with parameters $m_{n}$ and $p_{n}$, we have

$$
\lim _{n \rightarrow \infty} \mathrm{P}\left(C_{n}\right)=1, \quad \lim _{n \rightarrow \infty} \mathrm{P}\left(D_{n} \leq 7\left(\log \frac{n}{m_{n}+1}+1\right)\right)=1 .
$$

Conversely, if

$$
\begin{aligned}
& \frac{m_{n}}{n} \rightarrow 0 \text { as } n \rightarrow \infty, \text { and } \\
&\left(m_{n}+1\right) n p_{n}<(1-\delta)\left(\frac{m_{n}+1}{m_{n}+2}\right)^{2} \log \frac{n}{m_{n}+1}
\end{aligned}
$$

for some $\delta>0$ and infinitely many $n$, then there is a sequence of node locations such that $\liminf _{n \rightarrow \infty} \mathrm{P}\left(C_{n}\right)=0$.

Remarks. 1. If $m_{n}=0$ then Model A reduces to the classical Bernoulli model of Erdôs and Rényi. In this case our upper bound on the required node degree is conservative by a factor of 2 , and the lower bound by a factor of 4 . 
2. If $m_{n} \rightarrow \infty$ as $n \rightarrow \infty$ then the upper and lower bounds essentially differ by a factor of 2 .

3. Note that $m_{n}$ is the number of neighbours a node has in terms of the spatial graph, and $(n-1) p_{n}$ is the number of neighbours it has via shortcuts. Thus, the conditions of Theorem 1 state that the product of these quantities must be roughly equal to $\log n$ in order to ensure connectivity. For example, it suffices if $m_{n}=(1+\delta) \sqrt{2 \log n}$ and $n p_{n}=(1+\delta) \sqrt{2 \log n}$.

Theorem 2. Suppose that the sequences $r_{n}$ and $p_{n}$ are such that

$$
\pi r_{n}^{2}+n p_{n}=\log n+c_{n} \text { and } \lim _{n \rightarrow \infty} c_{n}=c .
$$

Then, the number of isolated nodes in the random graph generated by Model B with parameters $r_{n}$ and $p_{n}$ converges in distribution to a Poisson random variable with mean $\mathrm{e}^{-c}$. Moreover, if $\lim _{n \rightarrow \infty} c_{n}=-\infty$ then the random graph generated by Model B is disconnected w.h.p.

Remarks. 1 . Observe that $\pi r_{n}^{2}+n p_{n}$ is the mean node degree, so Theorem 2 states that the graph is disconnected if the mean node degree is much smaller than $\log n$, as is the case for both Bernoulli and spatial random graphs. Thus, in Model B there is no synergy between the nearest neighbour and shortcut links, at least as far as connectivity is concerned.

2. We recover the necessary condition for connectivity in spatial random graphs by setting $p_{n}=0$, and in Bernoulli random graphs by setting $r_{n}=0$.

3. The second claim of Theorem 2, which states that the random graph is disconnected w.h.p. if $c_{n} \rightarrow-\infty$, can be proved directly using the second moment method. Specifically, if $W$ denotes the number of isolated nodes in this random graph, we can use the inequality $\mathrm{P}(W=0) \leq \operatorname{var}(W) /(\mathrm{E} W)^{2}$, which follows from Chebyshev's inequality, to show that $\mathrm{P}(W=0) \rightarrow 0$. (The variance of $W$ can be bounded by bounding the probability that pairs of nodes are isolated.) It then follows that there is at least one isolated node and, hence, the graph is disconnected w.h.p. Instead, we shall obtain the result as a corollary of the first claim, which provides more detailed information regarding the number of isolated nodes. In particular, it shows that if $c_{n} \rightarrow c$ then the probability of being connected is asymptotically bounded by $\exp \left(-\mathrm{e}^{-c}\right)$.

\section{Proofs}

Proof of Theorem 1. Observe that under Model A, each node belongs to a connected component with at least $m_{n}+1$ nodes, since it is connected to $m_{n}$ nearest neighbours. The intuition behind the proof is that, for the graph to fail to be connected, there must be an isolated component of at least this size. The proof will proceed by reducing the small-world graph to a Bernoulli graph between clusters of size approximately $m_{n}$.

Given a graph $G$, we divide the nodes into disjoint groups as follows.

1. For each node $x$, let $C_{x}$ consist of $x$ and its $m_{n}$ nearest neighbours. We call $C_{x}$ the disc centred at $x$. Initially, the $\operatorname{discs} C_{x}$ are coloured black.

2. Considering the $n$ black discs in (any) sequence, colour each disc red if it does not intersect a disc already coloured red. If a disc $C_{x}$ becomes coloured red, we shall refer to it as the red disc centred at $x$. 
3. Pick the red discs in any sequence. Say the red disc centred at $x$ is chosen. Consider the nodes in all black discs overlapping it, if any. Group these nodes into disjoint sets of size $m_{n}+1$ and a residual set with $m_{n}$ or fewer nodes. Call each group of size $m_{n}+1 \mathrm{a}$ green disc, and absorb the $m_{n}$ or fewer residual nodes into the red disc. With some abuse of terminology, we shall refer to the (possibly) enlarged red disc and related green discs as all being centred at $x$.

The procedure terminates with nodes being grouped into disjoint discs $A_{k}, k=1,2, \ldots, K_{n}$, each of which is coloured either red or green. All green discs are of size $m_{n}+1$ and all red discs are of size between $m_{n}+1$ and $2 m_{n}+1$.

Observe that any two nodes $u$ and $v$ in the red disc centred at $x$ either come from the black disc that was centred at $x$ or they come from black discs that were centred at $y$ and $z$ and overlapped the black disc centred at $x$. (Possibly, $y=z$ or $y=x$ or $z=x$.) In the former case the graph distance (i.e. the number of edges along a shortest path) between nodes $u$ and $v$ in $G$ is at most equal to 2; in the latter case it is at most equal to 6 (since, if the discs centred at $x$ and $y$ intersect, there is a node $a$ which is a neighbour of both $x$ and $y$; thus, there is a path $u \rightarrow y \rightarrow a \rightarrow x \rightarrow b \rightarrow z \rightarrow v$ for some nodes $a$ and $b$ ). Either way, all nodes in the same red disc belong to the same connected component in $G$, considering only nearest-neighbour edges.

Likewise, any two nodes $u$ and $v$ belonging to the same green disc centred at $x$ come from black discs centred at $y$ and $z$ (possibly, $y=z$ ), which overlapped the black disc centred at $x$. Hence, these nodes belong to the same connected component in $G$ and are at most a graph distance 6 apart (there is a path $u \rightarrow y \rightarrow a \rightarrow x \rightarrow b \rightarrow z \rightarrow v$ for some nodes $a$ and $b$ ).

In order to show that $G$ is connected, it now suffices to show that the red and green discs form a connected graph when considering only shortcut edges. We shall do this by defining a Bernoulli graph $\tilde{G}$, as follows. Recall that each red disc has between $m_{n}+1$ and $2 m_{n}+1$ nodes. We first construct a subgraph $G_{1}$ of $G$ by deleting all but $m_{n}+1$ nodes in each red disc. (It does not matter which $m_{n}+1$ nodes are retained, so long as the choice is independent of the presence of edges in $G$. We can think of the edges of $G_{1}$ as being realised after the nodes are chosen.) In $G_{1}$ each disc, red or green, has exactly $m_{n}+1$ nodes, and if $G_{1}$ is connected then clearly so is $G$. Next, construct $\tilde{G}$ by replacing each disc $A_{k}$ in $G_{1}$ by a single node $k$, and putting an edge between nodes $j$ and $k$ if there is at least one shortcut edge between a node in $A_{j}$ and a node in $A_{k}$, in $G_{1}$. Clearly, $G_{1}$ is connected if $\tilde{G}$ is connected. But $\tilde{G}$ is a Bernoulli random graph on $K_{n}$ nodes, with edge probability

$$
\begin{aligned}
\tilde{p}_{n} & =1-\left(1-p_{n}\right)^{\left(m_{n}+1\right)^{2}} \\
& \geq 1-\exp \left(-\left(m_{n}+1\right)^{2} p_{n}\right) \\
& \geq 1-\exp \left(-2(1+\delta) \frac{m_{n}+1}{n} \log \frac{n}{m_{n}+1}\right),
\end{aligned}
$$

where the last inequality follows if (1) is assumed to hold. Moreover, $K_{n}$, the number of nodes in $\tilde{G}$, lies between $n /\left(2 m_{n}+1\right)$ and $n /\left(m_{n}+1\right)$. Hence, it follows, from (5), that

$$
\tilde{p}_{n} \geq 1-\exp \left(-\frac{(1+\delta) \log K_{n}}{K_{n}}\right) .
$$

Now, $K_{n} \rightarrow \infty$ as $n \rightarrow \infty$ by the assumption that $m_{n} / n \rightarrow 0$. Hence, from (6) we obtain

$$
\tilde{p}_{n} \geq\left(1+\delta^{\prime}\right) \frac{\log K_{n}}{K_{n}}
$$


for any $\delta^{\prime} \in(0, \delta)$ and all sufficiently large $n$. But $\tilde{p}_{n}$ is the edge probability in $\tilde{G}$, which is a Bernoulli random graph on $K_{n}$ nodes. Hence, by the results of Erdős and Rényi [5], $\mathrm{P}\left(C_{n}\right) \rightarrow 1$ as $n \rightarrow \infty$. This establishes the first claim in (2).

Moreover, from Theorem 10.17 of [3] it follows that the Bernoulli random graph $\tilde{G}$ on $K_{n}$ vertices satisfies

$$
\mathrm{P}\left(\operatorname{diameter}(\tilde{G}) \leq \frac{\log K_{n}+6}{\log \log K_{n}}+4\right) \rightarrow 1 \quad \text { as } K_{n} \rightarrow \infty .
$$

Since $K_{n} \leq n /\left(m_{n}+1\right)$, and $K_{n} \rightarrow \infty$ as $n \rightarrow \infty$, it follows that

$$
\mathrm{P}\left(\operatorname{diameter}(\tilde{G}) \leq \log \frac{n}{m_{n}+1}\right) \rightarrow 1 \quad \text { as } n \rightarrow \infty .
$$

Recall that each node in $\tilde{G}$ corresponds to a connected cluster in $G$, and that the diameter of this cluster is at most equal to 6 . Thus, $\operatorname{diameter}(G) \leq 7 \operatorname{diameter}(\tilde{G})+6$, and the second claim in (2) follows.

Conversely, consider a sequence $n_{k}, k \in \mathbb{N}$, such that (3) is satisfied along the sequence $n_{k}$, $m_{n_{k}}, p_{n_{k}}$ for some $\delta>0$. First, we argue that $n_{k}$ nodes can be partitioned into sets of size either $\tilde{m}_{n_{k}}+1$ or $\tilde{m}_{n_{k}}+2$, where the $\tilde{m}_{n_{k}}$ are such that $\left(\tilde{m}_{n_{k}}+1\right) /\left(m_{n_{k}}+1\right) \rightarrow 1$ as $k \rightarrow \infty$. To see this, write

$$
n_{k}=q\left(m_{n_{k}}+1\right)+r
$$

where $0 \leq r \leq m_{n_{k}}$, and $q$ and $r$ are integers. Now define $s=\lfloor r / q\rfloor$ to be the integer part of $r / q$, and let $b=r-q s$ and $a=q-b$. Then $a$ and $b$ are integers, $0 \leq b<q$ and $a>0$. Moreover, $r=q s+b=a s+b(s+1)$. Hence, we can rewrite (7) as

$$
n_{k}=a\left(m_{n_{k}}+s+1\right)+b\left(m_{n_{k}}+s+2\right),
$$

where $a+b=q$. Defining $\tilde{m}_{n_{k}}=m_{n_{k}}+s$, we see that $n_{k}$ nodes can be partitioned into $q$ sets, each of size $\tilde{m}_{n_{k}}+1$ or $\tilde{m}_{n_{k}}+2$.

Moreover, observe, from (7), that

$$
q \geq \frac{n_{k}}{m_{n_{k}}+1}-1, \quad \text { and so } \quad s \leq \frac{m_{n_{k}}\left(m_{n_{k}}+1\right)}{n_{k}-m_{n_{k}}-1} .
$$

Hence, by (3), $s /\left(m_{n_{k}}+1\right)$ tends to 0 as $k$ tends to $\infty$; consequently, $\left(\tilde{m}_{n_{k}}+1\right) /\left(m_{n_{k}}+1\right)$ tends to 1 , as claimed above.

We now consider the following deterministic sequence of node configurations. The configuration on $n_{k}$ nodes consists of clusters of size $\tilde{m}_{n_{k}}+1$ or $\tilde{m}_{n_{k}}+2$, where $\tilde{m}_{n_{k}}$ is defined as above. The nodes within each cluster are within Euclidean distance $\varepsilon_{n_{k}}$ of each other, and any two nodes in distinct clusters are more than $\varepsilon_{n_{k}}$ apart, for some positive constant $\varepsilon_{n_{k}}$. It is clear that such a $\varepsilon_{n_{k}}$ and node configuration can be found.

Denote the clusters by $A_{1}, A_{2}, \ldots, A_{q_{k}}$. There are clearly no nearest neighbour edges between clusters, only shortcuts. Let $G_{1}$ denote the graph on $q_{k}$ nodes obtained by replacing each cluster $A_{i}$ by a single node $i$, and putting an edge between nodes $i$ and $j$ only if there is a shortcut in $G$ between clusters $A_{i}$ and $A_{j}$. Clearly, $G$ is connected only if $G_{1}$ is connected (though the converse may not be true as the clusters $A_{i}$ may not be connected). Now, conditional on the cluster sizes, there is a shortcut (at least one) between clusters $A_{i}$ and $A_{j}$ with probability

$$
1-\left(1-p_{n_{k}}\right)^{\left|A_{i}\right|\left|A_{j}\right|} \leq \tilde{p}_{n_{k}}:=1-\left(1-p_{n_{k}}\right)^{\left(\tilde{m}_{n_{k}}+2\right)^{2}} .
$$


The presence of shortcuts between clusters are not independent events because the existence of one shortcut biases the conditional distribution of the size of the cluster and, thereby, the probability of other shortcuts from that cluster. Hence, $G_{1}$ is not a Bernoulli random graph. However, this problem is easily circumvented, as follows.

First, we augment each cluster of size $\tilde{m}_{n_{k}}+1$ in $G$ by adding a pseudonode which is within a distance $\varepsilon_{n_{k}}$ of all nodes in this cluster. Shortcuts are present between pseudonodes and other nodes with the same probability $p_{n_{k}}$ as for ordinary nodes, independent of the presence of other shortcuts. Call the augmented graph $\tilde{G}$. Now construct $\tilde{G}_{1}$ from $\tilde{G}$ analogous to how $G_{1}$ was constructed from $G$ : replace each cluster $A_{i}$ by a single node $i$, and put an edge between $i$ and $j$ in $\tilde{G}_{1}$ only if there is a shortcut between the (augmented) clusters $A_{i}$ and $A_{j}$ in $\tilde{G}$. It is clear from this construction that $\tilde{G}_{1}$ is a Bernoulli random graph on $q_{k}$ nodes with edge probability $\tilde{p}_{n_{k}}$ given by (8). Moreover, $G_{1}$ is a subgraph of $\tilde{G}_{1}$. Now $G$ is connected only if $G_{1}$ is connected, which in turn requires that $\tilde{G}_{1}$ be connected. We shall now use the result of Erdős and Rényi [5] to show that, w.h.p., $\tilde{G}$ fails to be connected.

Observe that $p_{n_{k}} \rightarrow 0$ as $k \rightarrow \infty$ by the assumption that $m_{n_{k}}$ and $p_{n_{k}}$ satisfy (3), i.e. that $n_{k}\left(m_{n_{k}}+1\right) p_{n_{k}}<\log n_{k}$. Hence, for any $\varepsilon>0$, we find, for all sufficiently large $k$, that the probability of a shortcut between two clusters is bounded by

$$
\tilde{p}_{n_{k}} \leq 1-\exp \left(-(1+\varepsilon)\left(\tilde{m}_{n_{k}}+2\right)^{2} p_{n_{k}}\right) \leq(1+\varepsilon)\left(\tilde{m}_{n_{k}}+2\right)^{2} p_{n_{k}} .
$$

Since the number of clusters $q_{k}$ lies in $\left[n_{k} /\left(\tilde{m}_{n_{k}}+2\right), n_{k} /\left(\tilde{m}_{n_{k}}+1\right)\right]$, using (3), we obtain

$$
\begin{aligned}
q_{k} \tilde{p}_{n_{k}} & \leq(1+\varepsilon) \frac{\left(\tilde{m}_{n_{k}}+2\right)^{2}}{\left(\tilde{m}_{n_{k}}+1\right)\left(m_{n_{k}}+1\right)}\left(m_{n_{k}}+1\right) n_{k} p_{n_{k}} \\
& \leq(1+\varepsilon)(1-\delta) \frac{\left(\tilde{m}_{n_{k}}+2\right)^{2}\left(m_{n_{k}}+1\right)}{\left(\tilde{m}_{n_{k}}+1\right)\left(m_{n_{k}}+2\right)^{2}} \log \frac{n_{k}}{m_{n_{k}}+1} .
\end{aligned}
$$

Now,

$$
\log \frac{n_{k}}{m_{n_{k}}+1}=\log \frac{n_{k}}{\tilde{m}_{n_{k}}+2}+\log \frac{\tilde{m}_{n_{k}}+2}{m_{n_{k}}+1} \leq(1+\varepsilon) \log q_{k}
$$

for all sufficiently large $k$, because $q_{k}$ tends to $\infty$ whereas $\left(\tilde{m}_{n_{k}}+1\right) /\left(m_{n_{k}}+1\right)$ tends to 1 , so that $\left(\tilde{m}_{n_{k}}+2\right) /\left(m_{n_{k}}+1\right)$ remains bounded as $k$ tends to $\infty$. Since $\varepsilon>0$ can be chosen arbitrarily small in $(9)$ and $\left(\tilde{m}_{n_{k}}+1\right) /\left(m_{n_{k}}+1\right)$ tends to 1 , it follows that

$$
q_{k} \tilde{p}_{n_{k}} \leq\left(1-\delta^{\prime}\right) \log q_{k}
$$

for any $\delta^{\prime} \in(0, \delta)$ and all sufficiently large $k$. Moreover, $q_{k} \rightarrow \infty$ as $k \rightarrow \infty$ by the assumption that $m_{n} / n \rightarrow 0$. Hence, using the results in [5] on the connectivity of Bernoulli random graphs, we find that $\mathrm{P}\left(\tilde{G}_{1}\right.$ is connected $) \rightarrow 0$ as $k \rightarrow \infty$. But $G$ is connected only if $\tilde{G}_{1}$ is connected. Therefore, $\mathrm{P}\left(C_{n_{k}}\right) \rightarrow 0$ as $k \rightarrow \infty$. This completes the proof.

Proof of Theorem 2. Let $W$ denote the number of isolated nodes in the graph generated by Model B with parameters $n, r_{n}$, and $p_{n}$. We do not make the dependence of $W$ on the parameters explicit in the notation. Let $Z$ denote a Poisson random variable with mean $\mathrm{E} W$. We use the Stein-Chen method to show that $W$ is close to $Z$ in total variation distance.

Let $U_{1}$ have the distribution of the number of isolated nodes, and let $1+V_{1}$ have the distribution of the number of isolated nodes conditional on node 1 being isolated. We shall construct $U_{1}$ and $V_{1}$ on the same probability space and show that $\mathrm{E}\left|U_{1}-V_{1}\right|$ is small. Then, 
we shall use [8, Chapter II, Theorem 24.3] to deduce that the total variation distance between $W$ and $Z$ is small.

The random variables $U_{1}$ and $V_{1}$ are constructed on the same probability space as follows. First, nodes 1 through $n$ are placed uniformly at random on the square $[-\sqrt{n} / 2, \sqrt{n} / 2]^{2}$, and the nearest neighbour and shortcut links are generated according to Model B. Next, all nodes located within a distance $r_{n}$ of node 1 (including node 1) are coloured red, while all other nodes are coloured green. Now, for each red node other than node 1 , we place an associated blue node uniformly at random in the portion of the square $[-\sqrt{n} / 2, \sqrt{n} / 2]^{2}$ which excludes the circle of radius $r_{n}$ centred at node 1 . (With some abuse of notation, we will use the same node label to refer to such associated nodes, distinguishing them by their colour.) The blue nodes carry the same shortcuts as the red nodes with which they are associated. In other words, if there is a shortcut between red nodes $i$ and $j$ (not equal to 1 ) then there is one between blue nodes $i$ and $j$, and likewise if there is a shortcut between red node $i$ and green node $j$. Finally, we put down nearest neighbour links between blue nodes and other blue or green nodes if they are within a distance $r_{n}$ of each other. Now, we define $U_{1}$ as the number of isolated nodes in the subgraph induced by red and green nodes, and $V_{1}$ as the number of isolated nodes in the subgraph induced by blue and green nodes. It is obvious that $U_{1}$ has the same distribution as $W$, the number of isolated nodes. To see that $1+V_{1}$ has the distribution of the number of isolated nodes conditional on node 1 being isolated, observe that, conditional on this event, the remaining nodes are uniformly distributed outside the circle of radius $r_{n}$ around node 1 , and that the shortcut distribution between these nodes is unchanged, while there are no shortcuts to node 1 . This is precisely the law of the subgraph on the blue and green nodes and, hence, the number of isolated nodes in this subgraph has the same distribution as $V_{1}$.

Let $U_{1}$ and $\mathcal{V}_{1}$ denote the set of isolated nodes in the red-green and blue-green subgraphs, respectively. Let $\mathcal{R}, \mathcal{B}$, and $\mathcal{G}$ respectively denote the set of red, blue, and green nodes, with respective cardinalities $R, B$, and $G$. In particular, $\mathcal{R}=\mathscr{B} \cup\{1\}$, and $\mathcal{R}$ and $g$ partition the node set. Now,

$$
\mathrm{E} W=\mathrm{E} U_{1}=\sum_{i=1}^{n} \mathrm{P}\left(i \in U_{1}\right)=n\left(1-\frac{\pi r_{n}^{2}}{n}\right)^{n-1}\left(1-p_{n}\right)^{n-1} \sim \exp \left(-c_{n}\right),
$$

where the last equivalence follows from (4). Moreover,

$$
\mathrm{E}\left[\left|U_{1}-V_{1}\right|\right] \leq \sum_{i=2}^{n} \mathrm{P}\left(i \in \mathcal{U}_{1}, i \notin \mathcal{V}_{1}\right)+\sum_{i=2}^{n} \mathrm{P}\left(i \notin \mathcal{U}_{1}, i \in \mathcal{V}_{1}\right) .
$$

If $i$ is one of the green nodes then, conditional on the event $\left\{i \in \mathcal{U}_{1}\right\}$, the event $\{i \notin$ $\left.\mathcal{V}_{1}\right\}$ can only occur if one of the blue nodes happens to fall within a distance $r_{n}$ of $i$; this happens independently for each blue node, with probability at most $\pi r_{n}^{2} /\left(n-\pi r_{n}^{2}\right)$. Moreover, conditional on $\left\{i \in \mathcal{U}_{1}\right\}$, the number of blue nodes is binomially distributed with parameters $n-2$ and $\pi r_{n}^{2} /\left(n-\pi r_{n}^{2}\right)$ if nodes 1 and $i$ are more than a distance $2 r_{n}$ apart; if they are less than $2 r_{n}$ apart, the number of blue nodes is stochastically dominated by such a binomial random variable. Hence,

$$
\mathrm{P}\left(i \notin \mathcal{V}_{1} \mid i \in \mathcal{U}_{1} \cap \mathcal{g}\right) \leq 1-\left(1-\frac{\pi^{2} r_{n}^{4}}{\left(n-\pi r_{n}^{2}\right)^{2}}\right)^{n-2} \leq \frac{\left(\log n+c_{n}\right)^{2}}{n}(1+o(1)) .
$$

We have used (4) to obtain the last inequality. Conversely, if $i \neq 1$ is one of the red nodes then the event $\left\{i \in \mathcal{U}_{1}\right\}$ cannot occur (as all the red nodes are neighbours of node 1, by definition). 
Thus, for $i \neq 1$, we have

$$
\begin{aligned}
\mathrm{P}\left(i \in \mathcal{U}_{1}, i \notin \mathcal{V}_{1}\right) & =\mathrm{P}\left(i \in \mathcal{U}_{1} \cap \mathcal{g}\right) \mathrm{P}\left(i \notin \mathcal{V}_{1} \mid i \in \mathcal{U}_{1} \cap \mathcal{g}\right) \\
& \leq \mathrm{P}\left(i \in \mathcal{U}_{1}\right) \mathrm{P}\left(i \notin \mathcal{V}_{1} \mid i \in U_{1} \cap g\right) \\
& \leq \frac{\left(\log n+c_{n}\right)^{2}}{n^{2}} \exp \left(-c_{n}\right)(1+o(1))
\end{aligned}
$$

where the last inequality is obtained using (10) and (12).

Next, if $i$ is a green node and $i \in \mathcal{V}_{1}$, then a necessary condition for the event $i \notin U_{1}$ to occur is that node $i$ either has a shortcut to node 1 , or node $i$ is at a distance between $r_{n}$ and $2 r_{n}$ from node 1 (so that it has a neighbour in the red-green subgraph, which is absent in the blue-green subgraph). Thus, for each green node $i$, we have

$$
\mathrm{P}\left(i \notin \mathcal{U}_{1}, i \in \mathcal{V}_{1} \cap g\right) \leq \mathrm{P}\left(\left\{i \in \mathcal{V}_{1} \cap g\right\} \cap A\right)+\mathrm{P}\left(\left\{i \in \mathcal{V}_{1} \cap g\right\} \cap B\right),
$$

where $A$ is the event that there is a shortcut between node $i$ and node 1 and $B$ is the event that node $i$ is at a distance between $r_{n}$ and $2 r_{n}$ from node 1 .

Any node $i \neq 1$ is isolated in the blue-green subgraph if it has no shortcuts to other blue or green nodes, and if there is no other node within a distance $r_{n}$ of it. Now, node $i$ is located at least a distance $r_{n}$ away from node 1 . Hence, at least half of the circle of radius $r_{n}$ centred around it lies outside the circle of radius $r_{n}$ centred around node 1, from which all nodes are moved out. Hence, the probability that a given node $j$ lies within a distance $r_{n}$ of node $i$ is at least $\pi r_{n}^{2} / 2\left(n-\pi r_{n}^{2}\right)$. Thus, we see that

$$
\mathrm{P}\left(i \in \mathcal{V}_{1} \mid A\right)=\mathrm{P}\left(i \in \mathcal{V}_{1}\right) \leq\left(1-p_{n}\right)^{n-2}\left(1-\frac{\pi r_{n}^{2}}{2\left(n-\pi r_{n}^{2}\right)}\right)^{n-2} .
$$

Hence,

$$
\begin{aligned}
\mathrm{P}\left(\left\{i \in \mathcal{V}_{1} \cap \mathcal{g}\right\} \cap A\right) & \leq \mathrm{P}(A) \mathrm{P}\left(i \in \mathcal{V}_{1} \mid A\right) \\
& \leq p_{n}\left(1-p_{n}\right)^{n-2}\left(1-\frac{\pi r_{n}^{2}}{2\left(n-\pi r_{n}^{2}\right)}\right)^{n-2} \\
& \leq \frac{p_{n} \exp \left(-c_{n} / 2\right)}{\sqrt{n}}[1+o(1)] \\
& \leq \exp \left(\frac{-c_{n}}{2}\right) n^{-3 / 2} \log n[1+o(1)] .
\end{aligned}
$$

The last two inequalities follow from (4). Likewise, we have

$$
\begin{aligned}
\mathrm{P}\left(\left\{i \in \mathcal{V}_{1} \cap g\right\} \cap B\right) & \leq \mathrm{P}(B) \mathrm{P}\left(i \in \mathcal{V}_{1} \mid B\right) \\
& \leq \frac{3 \pi r_{n}^{2}}{n-\pi r_{n}^{2}}\left(1-p_{n}\right)^{n-2}\left(1-\frac{\pi r_{n}^{2}}{2\left(n-\pi r_{n}^{2}\right)}\right)^{n-2} \\
& \leq \frac{3 \pi r_{n}^{2}}{n} \frac{\exp \left(-c_{n} / 2\right)}{\sqrt{n}}[1+o(1)] \\
& \leq 3 \exp \left(\frac{-c_{n}}{2}\right) n^{-3 / 2} \log n[1+o(1)] .
\end{aligned}
$$


Combining (14), (16), and (17), we obtain

$$
\mathrm{P}\left(i \notin \mathcal{U}_{1}, i \in \mathcal{V}_{1} \cap \mathcal{g}\right) \leq 4 \exp \left(\frac{-c_{n}}{2}\right) n^{-3 / 2} \log n[1+o(1)] .
$$

Conversely, if $i \neq 1$ is a red node then, automatically, $i$ is not isolated (in the red-green subgraph), so that

$$
\mathrm{P}\left(i \notin \mathcal{U}_{1}, i \in \mathcal{V}_{1} \cap \mathcal{R}\right)=\mathrm{P}\left(i \in \mathcal{V}_{1} \cap \mathcal{R}\right)=\mathrm{P}(i \in \mathcal{R}) \mathrm{P}\left(i \in \mathcal{V}_{1}\right) .
$$

Using (15), we obtain

$$
\begin{aligned}
\mathrm{P}\left(i \notin \mathcal{U}_{1}, i \in \mathcal{V}_{1} \cap \mathcal{R}\right) & \leq \frac{\pi r_{n}^{2}}{n}\left(1-\frac{\pi r_{n}^{2}}{2\left(n-\pi r_{n}^{2}\right)}\right)^{n-2}\left(1-p_{n}\right)^{n-2} \\
& \leq \frac{\log n+c_{n}}{n^{2}} \exp \left(-c_{n}\right)[1+o(1)] .
\end{aligned}
$$

Combining (18) and (19), we obtain

$$
\mathrm{P}\left(i \notin \mathcal{U}_{1}, i \in \mathcal{V}_{1}\right) \leq 4 \exp \left(\frac{-c_{n}}{2}\right) n^{-3 / 2} \log n[1+o(1)] .
$$

Finally, substituting (13) and (20) into (11) yields

$$
\mathrm{E}\left[\left|U_{1}-V_{1}\right|\right] \leq \frac{4 \log n}{\sqrt{n}} \exp \left(\frac{-c_{n}}{2}\right)[1+o(1)] .
$$

Recall that the number of isolated nodes, $W$, is the sum of Bernoulli random variables corresponding to the indicators that individual nodes are isolated, and that $Z$ is a Poisson random variable with the same mean as $W$. (The dependence of $W$ and $Z$ on $n$ has been suppressed in the notation.) Hence, by (10), (21), and [8, Chapter II, Theorem 24.3],

$$
d_{\mathrm{TV}}(W, Z) \leq 2\left(1 \wedge[\mathrm{E} W]^{-1}\right) \mathrm{E} W \mathrm{E}\left[\left|U_{1}-V_{1}\right|\right] \leq \frac{8 \log (n) \exp \left(-c_{n} / 2\right)}{\sqrt{n}}[1+o(1)],
$$

where $d_{\mathrm{TV}}(W, Z)$ denotes the total variation distance between the random variables $W$ and $Z$. Suppose that $c_{n} \rightarrow c$ and let $Y$ denote a Poisson random variable with mean $\mathrm{e}^{-c}$. Since $\mathrm{E} Z=\mathrm{E} W \sim \exp \left(-c_{n}\right)$, it is easy to see that $d_{\mathrm{TV}}(Z, Y)$ tends to 0 . By the triangle inequality, so does $d_{\mathrm{TV}}(W, Y)$. In other words, $W$ converges in distribution to a Poisson random variable with mean $\mathrm{e}^{-c}$ as $n$ tends to $\infty$. This establishes the first claim of the theorem.

To establish the second claim, first note that

$$
\mathrm{P}(W=0) \leq \mathrm{P}(Z=0)+d_{\mathrm{TV}}(W, Z)=\mathrm{e}^{-\mathrm{E} W}+d_{\mathrm{TV}}(W, Z),
$$

so that, by (10) and (22),

$$
\mathrm{P}(W=0) \leq\left(\exp \left(-\exp \left(-c_{n}\right)\right)+\frac{8 \log (n) \exp \left(-c_{n} / 2\right)}{\sqrt{n}}\right)[1+o(1)]
$$

In particular, if $c_{n}$ is fixed at $c$ then $\mathrm{P}(W=0) \rightarrow \exp \left(-\mathrm{e}^{-c}\right)$. Now, if $c_{n} \rightarrow-\infty$ then, for arbitrarily large negative $c$, we have $c_{n} \leq c$ for all large enough $n$. Moreover, if we augment the $n$th graph with additional random shortcuts so that $\pi r_{n}+n p_{n}=\log n+c$, then this does not reduce the number of isolated nodes. Hence, $\mathrm{P}(W=0) \leq 2 \exp \left(-\mathrm{e}^{-c}\right)$ say, for all sufficiently large $n$. Since $-c$ can be chosen arbitrarily large, $\mathrm{P}(W=0) \rightarrow 0$ as $n \rightarrow \infty$. In other words, there is at least one isolated node, and so the graph is disconnected, w.h.p. This completes the proof. 


\section{Conclusions and open problems}

We obtained a sufficient condition for connectivity in Model A and showed that this condition was necessary for a worst-case node configuration. It would be interesting to know whether the condition is tight (up to constants) for some random node configurations, such as the Poisson point process on a square. We have obtained necessary conditions for connectivity in Model B. While it is tempting to conjecture that there is a threshold for connectivity in this model at a mean degree of $\log n$, we do not have a proof of this result. Finally, the results here are obtained for a uniform shortcut distribution. The extension to random connection models where the probability of a shortcut between two nodes is some function of the Euclidean distance between them is an open problem.

\section{References}

[1] Balister, P., Bollobas, B., Sarkar, A. And Walters, M. (2005). Connectivity of random $k$-nearest-neighbour graphs. Adv. Appl. Prob. 37, 1-24.

[2] Barbour, A. D. and Reinert, G. (2001). Small worlds. Random Structures Algorithms 19, 54-74.

[3] Bollobas, B. (2001). Random Graphs. Cambridge University Press.

[4] Coppersmith, D., Gamarnik, D. And Sviridenko, M. (2002). The diameter of a long-range percolation graph. Random Structures Algorithms 21, 1-13.

[5] ERDős, P. AND RÉNYI, A. (1960). On the evolution of random graphs. Mat. Kutato Int. Közl. 5, $17-60$.

[6] Gupta, P. and Kumar, P. R. (1999). Critical power for asymptotic connectivity in wireless networks. In Stochastic Analysis, Control, Optimization and Applications, eds W. H. McEneaney et al., Birkhäuser, Boston, MA, pp. 547-566.

[7] Kleinberg, J. (2000). The small world phenomenon: an algorithmic perspective. In Proc. 32nd ACM Symp. Theory Comp. (STOC), ACM, New York, pp. 163-170.

[8] Lindvall, T. (1992). Lectures on the Coupling Method. Dover, New York.

[9] Meester, R. and Roy, R. (1996). Continuum Percolation. Cambridge University Press.

[10] Penrose, M. (1997). The longest edge of the random minimal spanning tree. Ann. Appl. Prob. 7, 340-361.

[11] Penrose, M. (2003). Random Geometric Graphs. Oxford University Press.

[12] Watts, D. J. (1999). Small Worlds. Princeton University Press.

[13] Xue, F. and Kumar, P. R. (2004). The number of neighbours needed for connectivity of wireless networks. Wireless Networks 10, 169-181. 